\title{
Association between Giardia and arthritis or joint pain in a large health insurance cohort: could it be reactive arthritis?
}

\author{
J. E. PAINTER ${ }^{1 *}$, S. A. COLLIER ${ }^{2}$ AND J. W. GARGANO ${ }^{2}$ \\ ${ }^{1}$ Department of Global and Community Health, College of Health and Human Services, George Mason \\ University, Fairfax, VA, USA \\ ${ }^{2}$ Division of Foodborne, Waterborne, and Environmental Diseases, National Center for Emerging Zoonotic and \\ Infectious Diseases, Centers for Disease Control and Prevention, Atlanta, GA, USA
}

Received 2 May 2016; Final revision 9 August 2016; Accepted 31 August 2016; first published online 19 September 2016

\section{SUMMARY}

This study aimed to assess the association between giardiasis and subsequent development of arthritis or joint pain using a retrospective cohort of individuals from a large administrative claims database in the United States. Using 2006-2010 data from MarketScan Commercial Claims and Encounters, we conducted a retrospective cohort study in people with an ICD-9-CM code for giardiasis $(n=3301)$ and persons without giardiasis $(n=14612)$ individually matched on age, sex, and enrolment length. We used conditional logistic regression to model the association between giardiasis and arthritis or joint pain documented in the 6 months following initial giardiasis diagnosis or index date for matched controls. After adjusting for healthcare utilization rate, giardiasis was associated with a $51 \%$ increase in claims for arthritis or joint pain (odds ratio $1 \cdot 51,95 \%$ confidence interval $1 \cdot 26-1 \cdot 80$ ). In age- and sex-stratified adjusted analyses, the association remained significant across all subgroups (age 0-19 years, age 20-64 years, males, and females). Findings from this study lend epidemiological support for the association between giardiasis and subsequent development of arthritis. Reactive arthritis might occur more frequently than has been reported in the literature. Further research is necessary to determine the mechanisms by which giardiasis could lead to arthritis.

Key words: Arthritis, commercial claims data, Giardia, giardiasis, medical claims, post-infectious arthritis, reactive arthritis.

\section{INTRODUCTION}

Giardiasis, an infection caused by the intestinal parasite Giardia intestinalis, is a major cause of human illness in the United States and worldwide [1-3]. Giardiasis causes substantial morbidity and mortality globally, particularly in areas with poor sanitation, institutions

\footnotetext{
* Author for correspondence: Dr J. E. Painter, PhD, MPH, Department of Global and Community Health, George Mason University, 4400 University Drive, Robinson Hall B432, Fairfax, VA 22030.

(Email: jpainte6@gmu.edu)
}

with children who are not toilet trained, or immunocompromised populations [3, 4]. An estimated $1 \cdot 2$ million cases occur annually in the United States, although fewer than 20000 cases are reported [2, 5].

Acute giardiasis is typically characterized by selflimited diarrhoea and intestinal symptoms (e.g. abdominal cramps, and malabsorption) [4, 6, 7]. However, additional symptoms have been documented to occur [8]. A recently published survey of non-outbreak giardiasis cases found that $33.8 \%$ reported at least one extraintestinal symptom, including symptoms of the eye, skin, urinary tract, or joint [8]. Furthermore, a 
review of published literature documenting long-term consequences of Giardia infections identified myriad post-infectious manifestations, including extraintestinal consequences such as ocular pathologies, allergies, muscular complications, and post-infectious arthritis [9].

The term 'reactive arthritis' was developed to describe arthritis that develops during or soon after an infection elsewhere in the body (e.g. in the gut or urogenital tract) $[10,11]$. Although diagnostic criteria vary, reactive arthritis is used to encompass a variety of rheumatological phenomena, such as inflammation of joints and other tissues, which develop during or soon after an infection [10,11]. While there is no unanimously accepted time-frame required for the diagnosis of reactive arthritis [10], various studies have used definitions requiring the onset of rheumatological symptoms ranging from $\geqslant 4$ days to $\leqslant 3$ months after gastrointestinal infection [10, 12-14]. The average duration of reactive arthritis is $3-5$ months [15]; however, chronic symptoms lasting at least 5 years after onset have been documented [16].

Incidence of reactive arthritis is difficult to estimate, as it depends largely on how it is defined, the epidemiological investigational methods used, and the associated pathogen [11]. Although the enteric pathogens classically linked to reactive arthritis include Yersinia, Salmonella, Campylobacter, and Shigella $[10,11,17-21]$, inflammatory arthritis has also been described following enteric infections with Clostridium difficile, Brucella, and Giardia [22]. Globally, the incidence of reactive arthritis is estimated to range from 1-30 cases/100 000 population [23]. In the United States, only one study has examined the incidence of reactive arthritis following bacterial enteric infections, including Yersinia, Salmonella, Campylobacter, Shigella, and E. coli $\mathrm{O} 157$ [17]. The estimated incidence was $0 \cdot 6-3 \cdot 1$ cases $/ 100000$ population; incidence was highest following Campylobacter and Salmonella infections.

Reactive arthritis or joint pain following Giardia has been documented in several case studies [24-27], case series [28, 29], and small research studies [8]. Despite the large burden of giardiasis in the Unites States and worldwide, the evidence supporting an association with reactive arthritis is limited [22], and the magnitude of post-giardiasis joint pain is unknown. To date, no large-scale epidemiological studies have examined the association between giardiasis and reactive arthritis. Here, we examine the association between giardiasis and the subsequent development of arthritis or joint pain using a retrospective cohort of individuals diagnosed with giardiasis in a large administrative claims database.

\section{METHODS}

Data were analysed from the Truven Health Analytics MarketScan Commercial Claims and Encounters database, an insurance database that captures medical billing data from a selection of large employers, health plans, and government and public organizations. This database includes active employees and their dependants, early (non-Medicare) retirees and dependants, and Consolidated Omnibus Budget Reconciliation Act (COBRA) continuees. Person-specific enrolment (i.e. signing up with a health insurance company rendering coverage effective), and clinical utilization claims (i.e. bills filed for medical services rendered) across inpatient and outpatient services were used.

We conducted a retrospective matched cohort study over the 5-year period from 1 January 2006 to 31 December 2010. We identified patients who incurred a claim that was assigned the International Classification of Diseases, Ninth Revision, Clinical Modification (ICD-9-CM) code 007.1 during the study period; these patients comprise the giardiasis cohort. The earliest record where a person was given this code was considered their index date; if a person was enrolled in the database more than one time, their diagnosis had to occur during their first enrolment to be included. Matched, unexposed patients (giardiasis-free group) were selected from the pool of patients who did not have an ICD-9-CM code for giardiasis, matched on sex, age (within 5-year age groups), and duration of enrolment in the database. Five unexposed patients were matched to each exposed person. For each person with giardiasis, we calculated the number of days from enrolment to giardiasis diagnosis. For each matched person without giardiasis, we added this number of days to their enrolment date and defined this date as their index date. Patients with the outcome of interest before their index date, patients who were enrolled in the database for $<90$ days before their index date, and patients with $<90$ days of follow-up after their index date were excluded from analyses. Thus, all cohort members were enrolled in the database for at least 6 consecutive months.

Reactive arthritis does not have a distinct ICD-9-CM code, so we examined this construct indirectly based on symptoms and timing. The main outcome variable was having a diagnostic code for arthritis or joint pain (hereafter referred to as joint pain) within 6 months after the 
index date. Given that previous studies have employed a window of up to 3 months for reactive arthritis to occur following enteric infection, combined with the potential for delay in seeking healthcare, we included claims made within 6 months of the index date as potential reactive arthritis cases. To create this variable, we included persons with an ICD-9-CM code of 711.00711.99 (arthropathies associated with infections); 714.0-714.9 (rheumatoid arthritis and other inflammatory polyarthropathies); 715.00-715.98 (osteoarthrosis and other allied disorders); 716.40-716.99 (other and unspecified arthropathies); 719.00-719.99 (other and unspecified joint disorders); or $729 \cdot 0$ (rheumatism) in their billing record within 6 months of their index date. The diagnostic code for Reiter's disease, a type of reactive arthritis affecting the eyes and urethra in addition to joints, $099 \cdot 3$, was also included.

Information on other patient descriptors was also obtained from the MarketScan data. Smoking, anxiety, and depression have been previously established as risk factors for arthritis [30-32], thus these were selected as comorbidities of interest and identified by ICD-9-CM codes [smoking (305.1, V15.82), anxiety (293.84, 300.00, 300.02, 300.09), and depression $(296 \cdot 2 x, 296 \cdot 3 x, 296 \cdot 82,300 \cdot 4,311)]$. We calculated a healthcare utilization rate by counting number of outpatient visits for any diagnosis in the time from enrolment to 90 days before index date, and dividing this count by the number of days between enrolment and 90 days before index date. We categorized this rate into quartiles for analysis. We considered patients to have received a laboratory diagnostic test for giardiasis if they had a procedure code claim for ova and parasite examination, direct fluorescent antibody test, enzyme immunoassay, immunofluorescent assay, or a cartridge test within 30 days before or after their first giardiasis diagnosis.

\section{Analysis}

Data were analysed using SAS v. 9.3 (SAS Institute Inc., USA). We assessed differences in demographics, outcome, and potentially confounding variables by giardiasis status. To preserve the matched study design, conditional logistic regression was used to model the association between giardiasis and joint pain occurring in the 6 months following the initial giardiasis diagnosis. Unadjusted logistic regression analyses were conducted to assess the association between giardiasis and each potentially confounding variable with joint pain. Variables that changed the odds ratio (OR) of giardiasis by $\geqslant 10 \%$ were included in the final multivariable model. Analyses were repeated with samples stratified by age and sex to explore potential effectmeasure modification by these matching variables.

\section{RESULTS}

Our initial matched cohort included 34918 patients $(n=5821$ patients in the giardiasis group, $n=29097$ patients in the non-giardiasis group). The final sample included 17913 patients $(n=3301$ in the giardiasis group, $n=14612$ in the non-giardiasis group) after excluding patients for the following reasons (exclusions were not mutually exclusive): patients with arthritis or joint pain documented before their index date ( $n=981$ in the giardiasis group, $n=3306$ in the nongiardiasis group); persons with $\leqslant 90$ days of enrolment before their index date $(n=1060$ in the giardiasis group, $n=5300$ in the non-giardiasis group), persons with $<90$ days of follow-up after their index date from analyses $(n=747$ in the giardiasis group, $n=$ 3742 in the non-giardiasis group), persons in the nongiardiasis group whose matched exposed person had a diagnosis of arthritis prior to their giardiasis diagnosis $(n=3861)$.

Age, sex, and duration of enrolment were similarly distributed in both groups as a result of the matched design (Table 1). There was a slight but statistically significant difference in the age-group composition of the two groups in spite of age matching, because after application of exclusion criteria, some persons in the giardiasis group were matched with fewer than five persons in the group without giardiasis. There were higher percentages of patients with medical claims for smoking, anxiety, and depression in the giardiasis group compared to the non-giardiasis group. There was also a higher percentage of patients in the highest healthcare utilization rate quartile in the giardiasis group compared to the non-giardiasis group. Of note, $17 \cdot 0 \%$ of patients in the giardiasis group had a medical claim for joint pain at any time following their index date, compared to $10.9 \%$ in the non-giardiasis group. The percentage of patients with joint pain in the 6 months after giardiasis diagnosis was higher in the giardiasis group compared to the non-giardiasis group, both overall and stratified by age group and sex.

In unadjusted analyses, the risk of having joint pain in the 6 months after giardiasis diagnosis was $69 \%$ higher in in the giardiasis group compared to the nongiardiasis group [OR $1 \cdot 69$, 95\% confidence interval 
Table 1. Patient characteristics by Giardia status, MarketScan commercial health insurance database, 2006-2010

\begin{tabular}{|c|c|c|c|c|c|}
\hline & \multicolumn{2}{|c|}{$\begin{array}{l}\text { No giardiasis } \\
(N=14612)\end{array}$} & \multicolumn{2}{|c|}{$\begin{array}{l}\text { Giardiasis } \\
(N=3301)\end{array}$} & \multirow{2}{*}{$\begin{array}{l}\chi^{2} \text { test } \\
P \text { value }\end{array}$} \\
\hline & $n$ & $(\%)$ & $n$ & $(\%)$ & \\
\hline \multicolumn{5}{|l|}{ Age, years } & $0 \cdot 014$ \\
\hline $0-4$ & 3032 & $(20 \cdot 8)$ & 621 & $(18 \cdot 8)$ & \\
\hline $5-9$ & 1298 & (8.9) & 272 & $(8 \cdot 2)$ & \\
\hline $10-14$ & 722 & $(4 \cdot 9)$ & 161 & $(4 \cdot 9)$ & \\
\hline $15-19$ & 850 & $(5 \cdot 8)$ & 191 & $(5 \cdot 8)$ & \\
\hline $20-39$ & 4255 & $(29 \cdot 1)$ & 949 & $(28 \cdot 8)$ & \\
\hline $40-64$ & 4457 & $(30 \cdot 5)$ & 1107 & $(33.5)$ & \\
\hline \multicolumn{5}{|l|}{ Sex } & 0.594 \\
\hline Male & 7724 & $(52 \cdot 9)$ & 1728 & $(52 \cdot 4)$ & \\
\hline Female & 6888 & $(47 \cdot 1)$ & 1573 & $(47 \cdot 7)$ & \\
\hline \multicolumn{5}{|l|}{ Duration of enrolment } & $0 \cdot 350$ \\
\hline 6 months -1 year & 2181 & $(14 \cdot 9)$ & 462 & $(14 \cdot 0)$ & \\
\hline$>1$ year -2 years & 3830 & $(26 \cdot 2)$ & 847 & $(25 \cdot 7)$ & \\
\hline$>2$ years -3 years & 2927 & $(20 \cdot 0)$ & 659 & $(20 \cdot 0)$ & \\
\hline$>3$ years -4 years & 2834 & $(19 \cdot 4)$ & 647 & $(19 \cdot 6)$ & \\
\hline$>4$ years -5 years & 2840 & $(19 \cdot 4)$ & 686 & $(20 \cdot 8)$ & \\
\hline \multicolumn{6}{|l|}{ Medical claims for: } \\
\hline Smoking & 411 & $(2 \cdot 8)$ & 153 & $(4 \cdot 6)$ & $<0 \cdot 001$ \\
\hline Anxiety & 695 & $(4 \cdot 8)$ & 335 & $(10 \cdot 2)$ & $<0 \cdot 001$ \\
\hline Depression & 840 & $(5 \cdot 8)$ & 346 & $(10 \cdot 5)$ & $<0 \cdot 001$ \\
\hline \multicolumn{5}{|l|}{ Healthcare utilization rate quartile } & $<0 \cdot 001$ \\
\hline 1 (lowest) & 4745 & $(32 \cdot 5)$ & 609 & $(18 \cdot 5)$ & \\
\hline 2 & 3832 & $(25 \cdot 9)$ & 731 & $(22 \cdot 1)$ & \\
\hline 3 & 3336 & $(22 \cdot 9)$ & 927 & $(28 \cdot 1)$ & \\
\hline 4 (highest) & 2699 & $(18 \cdot 5)$ & 1034 & $(31 \cdot 2)$ & \\
\hline Arthritis or joint pain & 1594 & $(10 \cdot 9)$ & 562 & $(17 \cdot 0)$ & $<0 \cdot 001$ \\
\hline \multicolumn{6}{|l|}{$\begin{array}{l}\text { Development of arthritis or joint pain } \\
\leqslant 6 \text { months of index date }\end{array}$} \\
\hline Overall & 507 & $(3 \cdot 5)$ & 196 & $(5 \cdot 9)$ & $<0 \cdot 001$ \\
\hline $0-19$ years & 101 & $(1.7)$ & 43 & $(3 \cdot 5)$ & $<0 \cdot 001$ \\
\hline 20-64 years & 406 & $(4 \cdot 7)$ & 153 & $(7 \cdot 4)$ & $<0 \cdot 001$ \\
\hline Males only & 243 & $(3 \cdot 2)$ & 95 & $(5 \cdot 5)$ & $<0 \cdot 001$ \\
\hline Females only & 264 & $(3 \cdot 8)$ & 101 & $(6 \cdot 4)$ & $<0 \cdot 001$ \\
\hline
\end{tabular}

(CI) 1.42-2.00] (Table 2). The risk of joint pain was also higher in patients with medical claims for smoking, anxiety, and depression. Compared to patients in the lowest healthcare utilization rate quartile, patients in the second, third, and fourth quartiles had a significantly higher risk of developing joint pain. The only variable which met the $10 \%$ change in estimate criterion for inclusion in the adjusted model was healthcare utilization rate quartile. In adjusted analyses, controlling for healthcare utilization rate attenuated the magnitude, but did not eliminate the association between giardiasis and joint pain (OR 1.51, 95\% CI 1.261.80). The association between arthritis and healthcare utilization rate also remained significant, particularly for patients in the third quartile (OR $1 \cdot 97,95 \%$ CI 1.51-2.59) and fourth quartile (OR $2 \cdot 40,95 \%$ CI $1 \cdot 84-3 \cdot 13)$. This final model was run again after excluding giardiasis patients who did not have evidence of receiving a diagnostic test, and results were not statistically different (not shown).

In unadjusted age-stratified analyses, the odds of joint pain was two times higher (i.e. OR 2.01) in persons with giardiasis in patients aged $0-19$ years and $61 \%$ higher in patients aged $20-64$ years (i.e. OR 1.61) (Table 3). Similarly, the odds of joint pain were higher in patients with giardiasis than without in both males and females. Controlling for healthcare utilization rate attenuated the OR for join pain for 
Table 2. Association between giardiasis and subsequent documentation of arthritis or joint pain within 6 months of giardiasis index date*, MarketScan commercial health insurance database, 2006-2010

\begin{tabular}{|c|c|c|c|c|}
\hline & \multicolumn{2}{|c|}{ Unadjusted } & \multicolumn{2}{|c|}{ Adjusted $\uparrow$} \\
\hline & OR & $(95 \% \mathrm{CI})$ & OR & $(95 \% \mathrm{CI})$ \\
\hline Giardiasis & 1.69 & $(1 \cdot 42-2 \cdot 00)$ & $1 \cdot 51$ & $(1 \cdot 26-1 \cdot 80)$ \\
\hline \multicolumn{5}{|c|}{ Medical claims for: } \\
\hline Smoking & 1.49 & $(1 \cdot 03-2 \cdot 16)$ & - & \\
\hline Anxiety & $2 \cdot 49$ & $(1 \cdot 88-3 \cdot 29)$ & - & \\
\hline Depression & 1.63 & $(1 \cdot 25-2 \cdot 13)$ & - & \\
\hline \multicolumn{5}{|c|}{$\begin{array}{l}\text { Healthcare utilization } \\
\text { rate quartile }\end{array}$} \\
\hline 1 & $1 \cdot 00$ & (ref.) & $1 \cdot 00$ & (ref.) \\
\hline 2 & $1 \cdot 41$ & $(1 \cdot 05-1 \cdot 88)$ & $1 \cdot 34$ & $(1 \cdot 00-1 \cdot 79)$ \\
\hline 3 & $2 \cdot 08$ & $(1 \cdot 59-2 \cdot 72)$ & 1.97 & $(1 \cdot 51-2 \cdot 59)$ \\
\hline 4 & $2 \cdot 67$ & $(2 \cdot 05-3 \cdot 46)$ & $2 \cdot 40$ & $(1 \cdot 84-3 \cdot 13)$ \\
\hline
\end{tabular}

OR, Odds ratio; CI, confidence interval; ref., referent.

* The earliest record where a patient was given the ICD-9 code for giardiasis was considered their index date. Matched, unexposed persons (non-giardiasis cohort) were assigned an index date.

$\dagger$ Adjusted for variables that change the risk ratio for giardiasis by $>10 \%$ in preliminary logistic regression analyses.

patients by giardiasis status in both age groups and both sexes; however, the associations remained significant in all subgroups. CIs around the ORs for these stratified analyses overlapped considerably.

\section{DISCUSSION}

This retrospective matched cohort study is unique in its examination of the association between giardiasis and joint pain and its use of a large administrative claims database, examining a broad segment of the US population. We identified a statistically significant $51 \%$ increased odds of receiving a diagnosis of joint pain during the 6 months following a giardiasis diagnosis. Previous studies identifying joint pain as a potential sequela of giardiasis have been relatively small and lacked a comparison group [9], including case studies [24-27], case series [28, 29], or small research studies [8]. Findings from this study lend epidemiological support for the association between giardiasis and subsequent development of joint pain identified in smaller uncontrolled studies [8, 9, 24-28].

Findings from this study indicate that giardiasis is epidemiologically associated with joint pain, in a timeframe that could plausibly be considered as reactive arthritis. Further, these findings suggest that
Table 3. Association between giardiasis and subsequent documentation of arthritis or joint pain within 6 months of giardiasis index date*, stratified by sex and age, MarketScan commercial health insurance database, 2006-2010

\begin{tabular}{|c|c|c|c|c|}
\hline & \multicolumn{2}{|c|}{$\begin{array}{l}\text { Unadjusted logistic } \\
\text { regression }\end{array}$} & \multicolumn{2}{|c|}{$\begin{array}{l}\text { Adjusted logistic } \\
\text { regression } \dagger\end{array}$} \\
\hline & $\mathrm{RR}$ & $(95 \% \mathrm{CI})$ & RR & $(95 \% \mathrm{CI})$ \\
\hline \multicolumn{5}{|l|}{ Age, years } \\
\hline 0-19 & $2 \cdot 01$ & $(1 \cdot 40-2 \cdot 88)$ & $1 \cdot 63$ & $(1 \cdot 12-2 \cdot 38)$ \\
\hline $20-64$ & $1 \cdot 61$ & $(1 \cdot 32-1 \cdot 95)$ & $1 \cdot 45$ & $(1 \cdot 19-1 \cdot 77)$ \\
\hline \multicolumn{5}{|l|}{ Sex } \\
\hline Male & 1.73 & $(1 \cdot 35-2 \cdot 21)$ & $1 \cdot 57$ & $(1 \cdot 22-2 \cdot 01)$ \\
\hline Female & 1.65 & $(1 \cdot 30-2 \cdot 09)$ & $1 \cdot 44$ & $(1 \cdot 12-1 \cdot 84)$ \\
\hline
\end{tabular}

RR, Risk ratio; CI, confidence interval.

* The earliest record where a patient was given the ICD-9 code for giardiasis was considered their index date. Matched, unexposed persons (non-giardiasis cohort) were assigned an index date.

$\dagger$ Adjusted for variables that change the RR for giardiasis by $>10 \%$ in preliminary logistic regression analyses; only healthcare utilization rate quartile met this criteria in both age and sex-stratified analyses.

extraintestinal sequelae to giardiasis may be more common than previously thought [8]. Joint pain associated with giardiasis may be more frequent than is reflected in the medical literature [28], given that the magnitude of risk for joint pain following a medical claim for giardiasis was higher than would be expected if there were no association with the outcome. Multiple studies have suggested that, while Giardia is a common cause of gastroenteritis [1-3], it is a relatively uncommon cause of reactive arthritis compared to other pathogens known to precede reactive arthritis [9, 25]. Based on the results of this study, we suspect that giardiasis could be an unrecognized or underestimated cause of joint pain. We found a $51 \%$ increase in the odds of joint pain in the giardiasis cohort compared to the non-giardiasis cohort, and the crude risk difference in onset of joint pain was $2 \cdot 4 \%$ (i.e. $5 \cdot 9-3 \cdot 5 \%$, see Table 1). If $2 \cdot 4 \%$ of the estimated 1.2 million annual US giardiasis cases develop joint pain, then up to 28800 people could be experiencing giardiasis-related joint pain annually in the United States. Although previous research has suggested that reactive arthritis associated with enteric infection should be considered in the differential diagnosis for undifferentiated arthritis [22], healthcare providers may fail to diagnose post-giardiasis joint pain for 
several reasons, perhaps in part because different healthcare providers could be involved in care for the two conditions.

Both giardiasis and arthritis vary by age and sex in the US population, and we were particularly interested in the implications of these demographic characteristics on the association between giardiasis and joint pain. Rates of reported giardiasis are higher in males compared to females and young children (age 0-9 years) compared to other age groups [2]. Older age and female sex are known risk factors for arthritis in general (non-reactive arthritis) [33]. Interestingly, the increase in risk for joint pain held true in analyses stratified by both age and sex, with a similar magnitude of increase in risk across strata of each and overlapping CIs.

There were higher percentages of patients with medical claims for smoking, anxiety, and depression in the giardiasis cohort compared to the non-giardiasis cohort. Smoking, anxiety, and depression have been previously established as risk factors for arthritis [30-32], and thus were considered as potential confounders of the association between giardiasis and arthritis or joint pain. Although smoking, anxiety, and depression were each significantly associated with arthritis or joint pain in unadjusted regression analyses, none impacted the association between giardiasis and arthritis or joint pain enough to be included in the final adjusted models. This finding held true in overall analyses, as well as analyses stratified by age and sex. This lends further support for a direct association between giardiasis and arthritis or joint pain.

This study has several limitations. Most importantly, this study used ICD-9-CM diagnostic codes from medical billing data, as opposed to medical records. While medical claims may approximate actual diagnoses, claims are an imperfect proxy for health states. In this case, we were interested in reactive arthritis. Because a specific code for arthritis subsequent to giardiasis does not exist, we used a collection of more general diagnosis codes for arthritis or joint pain. Validation studies comparing billing data to medical records for studying some types of arthritis exist [34, 35], but validation studies have not been published for giardiasis. Additionally, the MarketScan database only included patients with commercial medical insurance in the United States; thus findings may not be generalizable to populations without commercial medical insurance, or outside of the United States. While we identified a statistically significant association between medical claims for giardiasis and joint pain within a 6-month time-frame, we cannot conclude that the two were causally linked. Although increased medical care utilization is associated with receipt of an arthritis diagnosis [36], healthcare utilization rate did not fully explain the association between giardiasis and joint pain we observed; however, we cannot rule out the possibility that residual confounding due to healthcare-seeking behaviour underlies the observed association. Finally, additional known risk factors for arthritis, such as overweight and obesity, joint injuries, occupational exposures to repetitive joint motion, and bacterial infection [33], were not examined in this study. However, we do not think these are likely to be confounders because we have no reason to believe they are associated with the exposure (giardiasis). In the giardiasis cohort, comorbid diagnoses of Shigella, Salmonella, Campylobacter, E. coli, Cryptosporidium, C. difficile or norovirus infection were uncommon $(2 \cdot 2 \%)$; it is not plausible that such a small percentage of patients diagnosed with these infections is responsible for the $17 \cdot 0 \%$ of giardiasis patients who were diagnosed with arthritis.

This is the first large-scale analysis using medical claims data to examine the association between giardiasis and joint pain. Findings from this study provide epidemiological support for an association between giardiasis and joint pain in a small proportion of patients. Findings also suggest that reactive arthritis or joint pain may occur more frequently than reported. This has implications for studies of the burden of giardiasis, since for some patients, symptoms extend beyond the acute phase and might require treatment of extraintestinal manifestations. Further research is necessary to elucidate the clinical, epidemiological, and biological associations between giardiasis and arthritis or joint pain. Further research is also necessary to examine the possible role of early identification and treatment of giardiasis to reduce the occurrence of subsequent joint pain. Physicians who treat giardiasis, arthritis, and joint pain should consider the possibility of reactive arthritis following giardiasis infection.

\section{DECLARATION OF INTEREST}

None.

\section{REFERENCES}

1. Kappus KD, et al. Intestinal parasitism in the United States: update on a continuing problem. American Journal of Tropical Medicine and Hygiene 1994; 50: 705-713. 
2. Painter JE, et al. Giardiasis surveillance - United States, 2011-2012. Morbidity and Mortality Weekly Report. Surveillance Summaries 2015; 64 (Suppl. 3): 15-25.

3. Savioli L, Smith H, Thompson A. Giardia and Cryptosporidium join the 'Neglected Diseases Initiative'. Trends in Parasitology 2006; 22: 203-208.

4. Eberhard M, Gabrielli A, Savioli L. Giardiasis (Giardia enteritis). In: Heymann D, ed. Control of Communicable Diseases Manual, 19th edn. Washington, DC, 2008, pp. 258-260.

5. Scallan E, et al. Foodborne illness acquired in the United States - major pathogens. Emerging Infectious Diseases 2011; 17: 7-15.

6. Hellard ME, et al. Prevalence of enteric pathogens among community based asymptomatic individuals. Journal of Gastroenterology and Hepatology 2000; 15: 290-293.

7. Rodriguez-Hernandez J, Canut-Blasco A, Martin-Sanchez AM. Seasonal prevalences of Cryptosporidium and Giardia infections in children attending day care centres in Salamanca (Spain) studied for a period of 15 months. European Journal of Epidemiology 1996; 12: 291-295.

8. Cantey PT, et al. Study of nonoutbreak giardiasis: novel findings and implications for research. American Journal of Medicine 2011; 124: 1175.e1-1175.e8

9. Halliez MC, Buret AG. Extra-intestinal and long term consequences of Giardia duodenalis infections. World Journal of Gastroenterology 2013; 19: 8974-8985.

10. Townes JM. Reactive arthritis after enteric infections in the United States: the problem of definition. Clinical Infectious Diseases 2010; 50: 247-254.

11. Hannu T. Reactive arthritis. Best Practice \& Research Clinical Rheumatology 2011; 25: 347-357.

12. Simon DG, et al. Reiter's syndrome following epidemic shigellosis. Journal of Rheumatology 1981; 8: 969-973.

13. Lee AT, Hall RG, Pile KD. Reactive joint symptoms following an outbreak of Salmonella typhimurium phage type 135a. Journal of Rheumatology 2005; 32: 524-527.

14. McColl GJ, et al. HLA-B27 expression and reactive arthritis susceptibility in two patient cohorts infected with Salmonella Typhimurium. Australian and New Zealand Journal of Medicine 2000; 30: 28-32.

15. Hannu T, et al. Reactive arthritis or post-infectious arthritis? Best Practice \& Research Clinical Rheumatology 2006; 20: 419-433.

16. Thomson GT, et al. Post-Salmonella reactive arthritis: late clinical sequelae in a point source cohort. American Journal of Medicine 1995; 98: 13-21.

17. Townes JM, et al. Reactive arthritis following cultureconfirmed infections with bacterial enteric pathogens in Minnesota and Oregon: a population-based study. Annals of the Rheumatic Diseases 2008; 67: 1689-1696.

18. Hannu T, et al. Reactive arthritis after an outbreak of Yersinia pseudotuberculosis serotype O:3 infection. Annals of the Rheumatic Diseases 2003; 62: 866-869.

19. Hannu T, et al. Campylobacter-triggered reactive arthritis: a population-based study. Rheumatology 2002; 41: $312-318$.
20. Hannu T, et al. Reactive arthritis following an outbreak of Salmonella typhimurium phage type 193 infection. Annals of the Rheumatic Diseases 2002; 61: 264-266.

21. Hannu T, et al. Reactive arthritis attributable to Shigella infection: a clinical and epidemiological nationwide study. Annals of the Rheumatic Diseases 2005; 64: 594-598.

22. Hill Gaston JS, Lillicrap MS. Arthritis associated with enteric infection. Best Practice \& Research Clinical Rheumatology 2003; 17: 219-239.

23. Selmi C, Gershwin ME. Diagnosis and classification of reactive arthritis. Autoimmunity Reviews 2014; 13: 546-549.

24. Borman P, Seckin U, Ozoran K. Beaver fever - a rare cause of reactive arthritis. Journal of Rheumatology 2001; 28: 683.

25. Carlson DW, Finger DR. Beaver fever arthritis. Journal of Clinical Rheumatology: Practical Reports on Rheumatic \& Musculoskeletal Diseases 2004; 10: 86-88.

26. Shaw RA, Stevens MB. The reactive arthritis of giardiasis. A case report. Journal of the American Medical Association 1987; 258: 2734-2735.

27. Tupchong M, Simor A, Dewar C. Beaver fever - a rare cause of reactive arthritis. Journal of Rheumatology 1999; 26: 2701-2702.

28. Meza-Ortiz F. Giardiasis-associated arthralgia in children. Archives of Medical Research 2001; 32: 248-250.

29. Letts M, Davidson D, Lalonde F. Synovitis secondary to giardiasis in children. American Journal of Orthopedics 1998; 27: 451-454.

30. Kallberg H, et al. Smoking is a major preventable risk factor for rheumatoid arthritis: estimations of risks after various exposures to cigarette smoke. Annals of the Rheumatic Diseases 2011; 70: 508-511.

31. el-Miedany YM, el-Rasheed AH. Is anxiety a more common disorder than depression in rheumatoid arthritis? Joint Bone Spine 2002; 69: 300-306.

32. Patten SB, et al. Major depression as a risk factor for chronic disease incidence: longitudinal analyses in a general population cohort. Annals of General Hospital Psychiatry 2008; 30: 407-413.

33. Centers for Disease Control and Prevention. Arthritis: risk factors (http://www.cdc.gov/arthritis/basics/riskfactors.htm). Accessed May 2, 2016.

34. Carrara G, et al. A validation study of a new classification algorithm to identify rheumatoid arthritis using administrative health databases: case-control and cohort diagnostic accuracy studies. Results from the RECord linkage On Rheumatic Diseases study of the Italian Society for Rheumatology. BMJ Open 2015; 5: e006029.

35. Stringer E, Bernatsky S. Validity of juvenile idiopathic arthritis diagnoses using administrative health data. Rheumatology International 2015; 35: 575-579.

36. Lai NS, et al. Increased frequency and costs of ambulatory medical care utilization prior to the diagnosis of rheumatoid arthritis: a national population-based study. Arthritis Care \& Research 2014; 66: 371-378. 